\title{
Convex Hull Characterizations of Lexicographic Orderings
}

\author{
Warren Adams · Pietro Belotti · Ruobing \\ Shen
}

the date of receipt and acceptance should be inserted later

\begin{abstract}
Given a $p$-dimensional nonnegative, integral vector $\boldsymbol{\alpha}$, this paper characterizes the convex hull of the set $S$ of nonnegative, integral vectors $\boldsymbol{x}$ that is lexicographically less than or equal to $\boldsymbol{\alpha}$. To obtain a finite number of elements in $S$, the vectors $\boldsymbol{x}$ are restricted to be component-wise upper-bounded by an integral vector $\boldsymbol{u}$. We show that a linear number of facets is sufficient to describe the convex hull. For the special case in which every entry of $\boldsymbol{u}$ takes the same value $(n-1)$ for some integer $n \geq 2$, the convex hull of the set of $n$-ary vectors results. Our facets generalize the known family of cover inequalities for the $n=2$ binary case. They allow for advances relative to both the modeling of integer variables using base- $n$ expansions, and the solving of $n$-ary knapsack problems having weakly super-decreasing coefficients.
\end{abstract}

Keywords convex hull, facets, knapsack problem

\section{Introduction}

Given two vectors $\boldsymbol{\alpha}, \boldsymbol{u} \in \mathbb{Z}_{>\mathbf{0}}^{p}$, this paper computes the convex hull of the set of vectors $\boldsymbol{x} \in \mathbb{Z}_{\geq \mathbf{0}}^{p}$ having $\boldsymbol{x} \leq \boldsymbol{u}$ and $\boldsymbol{x}$ lexicographically less than or equal to $\boldsymbol{\alpha}$. Recalling that $\boldsymbol{x}$ is lexicographically less than or equal to $\boldsymbol{\alpha}$, denoted by $\boldsymbol{x} \preceq \boldsymbol{\alpha}$, if either $\boldsymbol{x}=\boldsymbol{\alpha}$ or the first nonzero entry of $\boldsymbol{x}-\boldsymbol{\alpha}$ is negative, this paper provides an explicit characterization of the convex hull of the set $S$, denoted by $\operatorname{conv}(S)$, where

$$
S \equiv\left\{\boldsymbol{x} \in \mathbb{Z}_{\geq \mathbf{0}}^{p}: \boldsymbol{x} \leq \boldsymbol{u}, \boldsymbol{x} \preceq \boldsymbol{\alpha}\right\}
$$

W. Adams

Department of Mathematical Sciences, Clemson University, Clemson, SC 29634

P. Belotti

Xpress Development Team, FICO, Birmingham B37 7GN, UK

R. Shen

Institut für Informatik, Universität Heidelberg, 69120 Heidelberg, Germany 
We make three assumptions, without loss of generality, followed by two remarks relative to the form of (1). First, we assume that $\boldsymbol{\alpha} \leq \boldsymbol{u}$ since, otherwise, if some $\alpha_{j}>u_{j}$, the problem reduces to finding lexicographic orderings over the first $j-1$ entries only, as fixing $x_{k}=u_{k}$ for all $k \geq j$ does not affect feasibility to (1). In this case, feasibility relative to the last $n-(j-1)$ integral entries of $\boldsymbol{x}$ is achieved by enforcing $0 \leq x_{k} \leq u_{k}$ for all $k \geq j$. Second, we assume that $\boldsymbol{u} \in \mathbb{Z}_{>\mathbf{0}}^{p}$ since $u_{j}=0$ for some $j$ fixes $x_{j}=0$ in all solutions. Third, we assume that $\alpha_{1}=u_{1}$ since every solution to (1) must have $x_{1} \leq \alpha_{1}$. Relative to remarks, we first note that the $\boldsymbol{x} \in \mathbb{Z}_{\geq \mathbf{0}}^{p}$ restrictions of (1) can be generalized to $\boldsymbol{x} \in \mathbb{Z}^{p}, \boldsymbol{x} \geq \boldsymbol{\ell}$ for some lower bounds $\boldsymbol{\ell} \in \mathbb{Z}^{p}$. Here, a substitution of variables $\boldsymbol{x}^{\prime}=\boldsymbol{x}-\boldsymbol{\ell}$ with $\boldsymbol{u}$ and $\boldsymbol{\alpha}$ adjusted to $\boldsymbol{u}^{\prime}=\boldsymbol{u}-\boldsymbol{\ell}$ and $\boldsymbol{\alpha}^{\prime}=\boldsymbol{\alpha}-\boldsymbol{\ell}$, respectively, affords the convex hull via $\boldsymbol{x}=\boldsymbol{x}^{\prime}+\boldsymbol{\ell}$. The first three assumptions are applicable to this transformed case so that $\boldsymbol{\alpha} \leq \boldsymbol{u}, \boldsymbol{u}>\boldsymbol{\ell}$, and $\alpha_{1}=u_{1}$. In addition, we need only consider those cases for which $\ell \leq \boldsymbol{\alpha}$ since if some $\ell_{j}>\alpha_{j}$, the problem reduces to finding lexicographic orderings over the first $j-1$ entries of $\boldsymbol{x}$. Our second remark is that the case where $\boldsymbol{x}$ is required to be lexicographically greater than or equal to a given $\boldsymbol{\eta}$, as opposed to $\boldsymbol{x} \preceq \boldsymbol{\alpha}$, can be reduced to the one in the present study by noticing that $\boldsymbol{x} \succeq \boldsymbol{\eta} \Leftrightarrow \boldsymbol{u}-\boldsymbol{x} \preceq \boldsymbol{u}-\boldsymbol{\eta}$. In this case, the first and third assumptions translate to $\boldsymbol{\eta} \geq \mathbf{0}$ and $\eta_{1}=0$, respectively, while the second assumption remains that $\boldsymbol{u} \in \mathbb{Z}_{>\mathbf{0}}^{p}$.

Observe that for the special case in which each entry $u_{j}$ of $\boldsymbol{u}$ has $u_{j}=n-1$ for some integer $n$, the problem is that of computing the convex hull of the set of $n$-ary vectors that is lexicographically less than or equal to $\boldsymbol{\alpha}$. Such sets $S$ of (1) have relevance in computing base- $n$ expansions of integer variables. To see this, suppose that we have a nonnegative integer variable $y$ that is bounded above by some scalar $\beta$, where $n^{p-1} \leq \beta<n^{p}$. Further suppose that the vector $\boldsymbol{\alpha} \in \mathbb{Z}_{>\mathbf{0}}^{p}$ with $\alpha_{j} \leq n-1$ for all $j$ is subsequently defined in terms of $\beta$ so that $\beta=\sum_{j=1}^{p} \bar{n}^{p-j} \alpha_{j}$, with $\boldsymbol{u}$ given by $u_{j}=n-1$ for all $j$ (and $u_{1}=\alpha_{1}$ due to the third assumption above). Then the $n$-ary expansion of $y$ computed by the set:

$$
T \equiv\left\{(\boldsymbol{x}, y) \in \mathbb{Z}_{\geq \mathbf{0}}^{p} \times \mathbb{R}^{1}: \boldsymbol{x} \leq \boldsymbol{u}, y=\sum_{j=1}^{p} n^{p-j} x_{j}, y \leq \beta\right\},
$$

is equivalently expressed by the set:

$$
W \equiv\left\{(\boldsymbol{x}, y) \in \mathbb{R}^{p} \times \mathbb{R}^{1}: y=\sum_{j=1}^{p} n^{p-j} x_{j}, \boldsymbol{x} \in S\right\} .
$$

Consequently, an explicit representation of $\operatorname{conv}(S)$ gives $\operatorname{conv}(T)$, as:

$$
\operatorname{conv}(T)=\operatorname{conv}(W)=\left\{(\boldsymbol{x}, y) \in \mathbb{R}^{p} \times \mathbb{R}^{1}: y=\sum_{j=1}^{p} n^{p-j} x_{j}, \boldsymbol{x} \in \operatorname{conv}(S)\right\} .
$$

Now, given $\boldsymbol{\alpha} \in \mathbb{Z}_{\geq \mathbf{0}}^{p}$ and $\boldsymbol{u} \in \mathbb{Z}_{>\mathbf{0}}^{p}$ with $\boldsymbol{\alpha} \leq \boldsymbol{u}$ and $\alpha_{1}=u_{1}$, a vector $\boldsymbol{x} \in \mathbb{Z}_{\geq \mathbf{0}}^{p}$ with $\boldsymbol{x} \leq \boldsymbol{u}$ will have $\boldsymbol{x} \in S$ if and only if the following condition is satisfied: for each $i \in\{2, \ldots, p\}$ with $x_{i}>\alpha_{i}$, there must exist a $j<i$ so that $x_{j}<\alpha_{j}$. Equivalently, we must have:

$$
\sum_{j=1}^{i-1}\left(\max \left\{0, \alpha_{j}-x_{j}\right\}\right) \geq 1 \text { for all } i \in\{2, \ldots, p\} \text { such that } x_{i}>\alpha_{i} .
$$


Of course, inequality (2) is not needed for any $i$ having $\alpha_{i}=u_{i}$. Furthermore, for the special case in which $\boldsymbol{u}=\mathbf{1}$ and $\boldsymbol{\alpha}$ is binary-valued so that each $x_{i}$ is restricted to be binary, (2) simplifies to the minimal cover inequalities:

$$
\sum_{j<i: \alpha_{j}=1}\left(1-x_{j}\right) \geq x_{i} \text { for all } i \in\{2, \ldots, p\} \text { such that } \alpha_{i}=0
$$

This special case of $S$ having $\boldsymbol{u}=\mathbf{1}$ and $\boldsymbol{\alpha}$ binary-valued has been extensively studied relative to both lexicographic orderings and specially-structured 0-1 knapsack polytopes having weakly super-decreasing coefficients. The works $[2,3,5,8]$ collectively characterize the set $S$, showing that the minimal cover inequalities of (3), together with the bounding restrictions $\mathbf{0} \leq \boldsymbol{x} \leq \mathbf{1}$, are sufficient to define the convex hull. A key observation is that inequalities (3) possess the "interval matrix" (consecutive ones) property (see [7, page 544, Definition 2.2] or [10]). Moreover, $[1,6]$ describe the convex hull of the set of binary vectors that is lexicographically lower and upper bounded by binary vectors.

This paper, which is an update of preliminary work found in [9], extends earlier contributions relative to lexicographic upper bounds by allowing nonnegative integer variables with general upper bounds. A recent paper [4] also considers these more general bounds, and independently offers an alternate convex hull argument. Our approach differs from this related work in that we identify and exploit a characterization of the constraint matrix to obtain nonsingular linear transformations between our spaces and known integral polytopes. As will be shown, the inequalities and arguments needed to characterize $\operatorname{conv}(S)$, and consequently $\operatorname{conv}(W)$, are different from (3), but reduce to this simpler form when $\boldsymbol{\alpha} \leq \boldsymbol{u}=\mathbf{1}$.

\section{Characterization of Valid Inequalities}

Given $\boldsymbol{\alpha} \in \mathbb{Z}_{\geq \mathbf{0}}^{p}$ and $\boldsymbol{u} \in \mathbb{Z}_{>\mathbf{0}}^{p}$ with $\boldsymbol{\alpha} \leq \boldsymbol{u}$ and $\alpha_{1}=u_{1}$, we begin by establishing properties of inequalities of the form $\sum_{j=1}^{p} \gamma_{j} x_{j} \leq \beta$ that are valid for $S$.

Lemma 1 Given any inequality of the form $\sum_{j=1}^{p} \gamma_{j} x_{j} \leq \beta$ that is valid for $S$, the inequality $\sum_{j=1}^{p} \max \left\{0, \gamma_{j}\right\} x_{j} \leq \beta$ is also valid for $S$.

Proof Consider any inequality of the form $\sum_{j=1}^{p} \gamma_{j} x_{j} \leq \beta$ that is valid for $S$, and having an index $t$ with $\gamma_{t}<0$. For each $\overline{\boldsymbol{x}} \in S$, the vector $\hat{\boldsymbol{x}}$ defined in terms of $\overline{\boldsymbol{x}}$ by $\hat{x}_{j}=\bar{x}_{j}$ for all $j \neq t$ and $\hat{x}_{t}=0$ has $\hat{\boldsymbol{x}} \in S$ so that $0 \bar{x}_{t}+\sum_{j \neq t} \gamma_{j} \bar{x}_{j}=\sum_{j=1}^{p} \gamma_{j} \hat{x}_{j} \leq \beta$.

By virtue of the above Lemma, and since $\boldsymbol{x} \geq \mathbf{0}$, we henceforth assume that $\gamma_{j} \geq 0$ for all $j$. Notably, every valid inequality $\sum_{j=1}^{p} \gamma_{j} x_{j} \leq \beta$ for $S$ must have $\beta \geq \sum_{j=1}^{p} \gamma_{j} \alpha_{j}$ since $\alpha \in S$. We direct attention to those inequalities with $\beta=$ $\sum_{j=1}^{p} \gamma_{j} \alpha_{j}$. In fact, we will show that a family of $p$ such inequalities, together with the $\mathbf{0} \leq \boldsymbol{x} \leq \boldsymbol{u}$ restrictions, provides $\operatorname{conv}(S)$. Accordingly, consider Theorem 1 below. 
Theorem 1 Given $\boldsymbol{\alpha} \in \mathbb{Z}_{\geq \mathbf{0}}^{p}$ and $\boldsymbol{u} \in \mathbb{Z}_{>\mathbf{0}}^{p}$ with $\boldsymbol{\alpha} \leq \boldsymbol{u}$ and $\alpha_{1}=u_{1}$, an inequality of the form:

$$
\sum_{j=1}^{p} \gamma_{j}\left(\alpha_{j}-x_{j}\right) \geq 0
$$

with $\gamma_{j} \geq 0$ for all $j \in\{1, \ldots, p\}$ is valid for $S$ if and only if:

$$
\gamma_{j} \geq \sum_{k=j+1}^{p} \gamma_{k}\left(u_{k}-\alpha_{k}\right) \forall j \in\{1, \ldots, p-1\} \text { such that } \alpha_{j} \neq 0 .
$$

Proof (Only if) Consider any inequality of the form (4) with $\gamma_{j} \geq 0$ for all $j \in$ $\{1, \ldots, p\}$ that is valid for $S$, and select any $t \in\{1, \ldots, p-1\}$ having $\alpha_{t} \neq 0$. The vector $\overline{\boldsymbol{x}} \in S$ given by $\bar{x}_{j}=\alpha_{j}$ for $j<t, \bar{x}_{t}=\alpha_{t}-1$, and $\bar{x}_{j}=u_{j}$ for $j>t$ has:

$$
\gamma_{t}+\sum_{j=t+1}^{p} \gamma_{j}\left(\alpha_{j}-u_{j}\right) \geq 0
$$

when inserted into (4), verifying (5).

(If) Consider any inequality of the form (4) with $\gamma_{j} \geq 0$ for all $j \in\{1, \ldots, p\}$ that satisfies (5). Arbitrarily select $\overline{\boldsymbol{x}} \in S$. The proof is to show that $\overline{\boldsymbol{x}}$ satisfies (4). If $\overline{\boldsymbol{x}}=\boldsymbol{\alpha}$, then (4) is trivially satisfied. Otherwise, let $t$ be the first entry of $\overline{\boldsymbol{x}}$ having $\bar{x}_{j} \neq \alpha_{j}$, so that $\bar{x}_{t}<\alpha_{t} \neq 0$ as $\overline{\boldsymbol{x}} \in S$. Then:

$$
\sum_{j=1}^{p} \gamma_{j}\left(\alpha_{j}-\bar{x}_{j}\right)=\gamma_{t}\left(\alpha_{t}-\bar{x}_{t}\right)+\sum_{j>t} \gamma_{j}\left(\alpha_{j}-\bar{x}_{j}\right) \geq \gamma_{t}+\sum_{j>t} \gamma_{j}\left(\alpha_{j}-u_{j}\right) \geq 0
$$

where the equality results from $\bar{x}_{j}=\alpha_{j}$ for all $j<t$, the left inequality follows from the nonnegativity of $\gamma$, together with $\bar{x}_{t} \leq \alpha_{t}-1$ and $\bar{x}_{j} \leq u_{j}$ for all $j>t$, and the right inequality follows from (5). This completes the proof.

Now consider a particular family of $p$ inequalities of (4) that is defined relative to (5) in the following manner. For each $i \in\{1, \ldots, p\}$ and each $j \leq i$, let $\gamma_{i j}$ denote that coefficient in inequality $i$ associated with the expression $\left(\alpha_{j}-x_{j}\right)$, and set all coefficients on expressions $\left(\alpha_{j}-x_{j}\right)$ with $j>i$ to 0 . Then, for each $i$, set $\gamma_{i i}=1$, and recursively define the $(i-1)$ coefficients $\gamma_{i j}$ having $j<i$, proceeding from $j=i-1$ to $j=1$, so that (5) is satisfied with equality when $\alpha_{j} \neq 0$ and $\gamma_{i j}=0$ when $\alpha_{j}=0$. Summarizing, we have:

$$
\gamma_{i j}=\left\{\begin{array}{ll}
1 & \text { if } j=i \\
0 & \text { if } j<i, \alpha_{j}=0 \\
\sum_{k=j+1}^{i} \gamma_{i k}\left(u_{k}-\alpha_{k}\right) & \text { if } j<i, \alpha_{j} \neq 0
\end{array} \quad \forall(i, j), i \geq j .\right.
$$

Construct a new set $F$ by replacing the $\boldsymbol{x} \preceq \boldsymbol{\alpha}$ restrictions in $S$ of (1) by these $p$ inequalities to obtain:

$$
F \equiv\left\{\boldsymbol{x} \in \mathbb{Z}_{\geq \mathbf{0}}^{p}: \boldsymbol{x} \leq \boldsymbol{u}, \sum_{j \leq i} \gamma_{i j}\left(\alpha_{j}-x_{j}\right) \geq 0 \text { for all } i \in\{1, \ldots, p\}\right\} .
$$

Then we have the following result. 
Lemma 2 The sets $S$ and $F$ found in (1) and (7), respectively, have $S=F$.

Proof The containment $S \subseteq F$ holds true since the $p$ inequalities found within (7) but not (1) are valid for $S$ by Theorem 1 . To show that $F \subseteq S$, consider any $\overline{\boldsymbol{x}} \in F$. If $\overline{\boldsymbol{x}}=\boldsymbol{\alpha}$, then $\overline{\boldsymbol{x}} \in S$. Otherwise, let $t$ be the first entry of $\overline{\boldsymbol{x}}$ having $\bar{x}_{j} \neq \alpha_{j}$. Then:

$$
\alpha_{t}-\bar{x}_{t}=\gamma_{t t}\left(\alpha_{t}-\bar{x}_{t}\right)=\sum_{j \leq t} \gamma_{t j}\left(\alpha_{j}-\bar{x}_{j}\right) \geq 0
$$

where the first equality is due to the definition of $\gamma_{t t}=1$ in (6), the second equality results from $\alpha_{j}=\bar{x}_{j}$ for all $j<t$, and the inequality follows from (7) with $i=t$. Thus, $\bar{x}_{t}<\alpha_{t}$, and the proof is complete.

Remark 1 Within (7), for each $i$ such that $\alpha_{i}=u_{i}$, we have $\gamma_{i j}=0$ for all $j<i$ so that the associated inequality reduces to the upper bounding restriction $u_{i}-x_{i} \geq 0$. This simplification is consistent with the observation that (2) is not needed when $\alpha_{i}=u_{i}$ (provided that $x_{i} \leq u_{i}$ is enforced). As a consequence, each such inequality $\sum_{j \leq i} \gamma_{i j}\left(\alpha_{j}-x_{j}\right) \geq 0$, by assumption including that inequality having $i=1$, implies the associated upper bounding restriction on $x_{i}$.

The below example illustrates the relationship between the sets $S$ and $F$ described in Lemma 2 and Remark 1.

Example 1 Let $p=5$ and $\boldsymbol{\alpha}$ and $\boldsymbol{u}$ be defined so that $\boldsymbol{\alpha}^{T}=(3,4,1,0,2)$ and $\boldsymbol{u}^{T}=(3,4,5,3,4)$. The lower-triangular $5 \times 5$ matrix $\left[\begin{array}{ccccc}1 & 0 & 0 & 0 & 0 \\ 0 & 1 & 0 & 0 & 0 \\ 4 & 4 & 1 & 0 & 0 \\ 15 & 15 & 3 & 1 & 0 \\ 10 & 10 & 2 & 0 & 1\end{array}\right]$ has the $(i, j)^{t h}$ entry recording $\gamma_{i j}$ from (6) for each $(i, j), i \geq j$. The set $F$ of $(7)$ becomes, in matrix notation:

$$
F \equiv\left\{\boldsymbol{x} \in \mathbb{Z}_{\geq \mathbf{0}}^{5}: \boldsymbol{x} \leq \boldsymbol{u},\left[\begin{array}{ccccc}
1 & 0 & 0 & 0 & 0 \\
0 & 1 & 0 & 0 & 0 \\
4 & 4 & 1 & 0 & 0 \\
15 & 15 & 3 & 1 & 0 \\
10 & 10 & 2 & 0 & 1
\end{array}\right]\left[\begin{array}{l}
\left(3-x_{1}\right) \\
\left(4-x_{2}\right) \\
\left(1-x_{3}\right) \\
\left(0-x_{4}\right) \\
\left(2-x_{5}\right)
\end{array}\right] \geq\left[\begin{array}{l}
0 \\
0 \\
0 \\
0 \\
0
\end{array}\right]\right\}
$$

which defines the same set of integral points $\boldsymbol{x}$ as $S$ of (1). Since $\alpha_{1}=u_{1}=3$ and $\alpha_{2}=u_{2}=4$, the first and second inequalities enforce that $3-x_{1} \geq 0$ and $4-x_{2} \geq 0$, respectively, as noted in Remark 1 .

Because Lemma 2 established that $S=F$, it immediately follows that $\operatorname{conv}(S)=$ $\operatorname{conv}(F)$. We show in the next section that the polytope obtained by relaxing the $\boldsymbol{x} \in \mathbb{Z}_{\geq \mathbf{0}}^{p}$ restrictions in $F$ of $(7)$ to $\boldsymbol{x} \in \mathbb{R}_{\geq \mathbf{0}}^{p}$ to obtain:

$$
\bar{F} \equiv\left\{\boldsymbol{x} \in \mathbb{R}_{\geq \mathbf{0}}^{p}: \boldsymbol{x} \leq \boldsymbol{u}, \sum_{j \leq i} \gamma_{i j}\left(\alpha_{j}-x_{j}\right) \geq 0 \text { for all } i \in\{1, \ldots, p\}\right\}
$$

has all integer extreme points, giving us that $\bar{F}=\operatorname{conv}(F)$ so that $\bar{F}=\operatorname{conv}(S)$.

Before concluding this section, we provide in Lemma 3 below an alternate description of the coefficients $\gamma_{i j}$ defined in (6). This description will allow us to characterize inverses of matrices whose entries consist of special subsets of the scalars $\gamma_{i j}$; these inverses are a key ingredient to our convex hull argument found in the proof of the upcoming Theorem 2. 
Lemma 3 The coefficients $\gamma_{i j}$ defined in (6) are equivalent to:

$$
\gamma_{i j}=\left\{\begin{array}{ll}
1 & \text { if } j=i \\
0 & \text { if } j<i, \alpha_{j}=0 \\
\left(u_{i}-\alpha_{i}\right) \prod_{\substack{k=j \neq 1: \\
\alpha_{k} \neq 0}}^{i-1}\left(1+u_{k}-\alpha_{k}\right) & \text { if } j<i, \alpha_{j} \neq 0
\end{array} \quad \forall(i, j), i \geq j,\right.
$$

where $\prod_{\substack{k=j+1: \\ \alpha_{k} \neq 0}}^{i-1}\left(1+u_{k}-\alpha_{k}\right)=1$ for products over null sets.

Proof The proof is to show for each $(i, j), j<i, \alpha_{j} \neq 0$, that the values of $\gamma_{i k}$ for $k \in\{j+1, \cdots, i\}$ defined in (6) have:

$$
\sum_{k=j+1}^{i} \gamma_{i k}\left(u_{k}-\alpha_{k}\right)=\left(u_{i}-\alpha_{i}\right) \prod_{\substack{k=j+1: \\ \alpha_{k} \neq 0}}^{i-1}\left(1+u_{k}-\alpha_{k}\right)
$$

as the left expression is $\gamma_{i j}$ by (6). Arbitrarily select an $i \geq 2$ and accordingly define $J \equiv\left\{j: j \leq i-1\right.$ and $\left.\alpha_{j} \neq 0\right\}$ to denote the set of indices less than $i$ with positive $\alpha_{j}$. (By assumption, $\alpha_{1} \neq 0$ so $J \neq \emptyset$.) Arrange the elements of $J=\left\{j_{1}, \cdots, j_{|J|}\right\}$ in increasing order so that $1=j_{1}<\cdots<j_{|J|}$. When $j=j_{|J|}$, we have:

$$
\sum_{k=j_{|J|}+1}^{i} \gamma_{i k}\left(u_{k}-\alpha_{k}\right)=u_{i}-\alpha_{i}=\left(u_{i}-\alpha_{i}\right) \prod_{\substack{k=j_{|J|}+1: \\ \alpha_{k} \neq 0}}^{i-1}\left(1+u_{k}-\alpha_{k}\right)
$$

where the first equation follows from (6) since $\gamma_{i k}=0$ for all $k \in\left\{j_{|J|}+1, \cdots, i-\right.$ $1\}$ and since $\gamma_{i i}=1$, and where the second equation trivially follows from the definition within Lemma 3 that products over null sets equal to 1 . Then (10) holds for the chosen $i$ when $j=j_{|J|}$, with $\gamma_{i j_{|J|}}=\left(u_{i}-\alpha_{i}\right)$, and so we consider the case where $i$ has $|J| \geq 2$.

When $|J| \geq 2$, then (6) enforces:

$$
\gamma_{i j_{p}}-\gamma_{i j_{p+1}}=\gamma_{i j_{p+1}}\left(u_{j_{p+1}}-\alpha_{j_{p+1}}\right) \text { for each } j_{p} \in J, p \neq|J|,
$$

or, equivalently, that:

$$
\gamma_{i j_{p}}=\gamma_{i j_{p+1}}\left(1+u_{j_{p+1}}-\alpha_{j_{p+1}}\right) \text { for each } j_{p} \in J, p \neq|J| \text {. }
$$

Then we obtain from (11), via induction on $p$ with $\gamma_{i j_{|J|}}=\left(u_{i}-\alpha_{i}\right)$, that $(6)$ enforces:

$$
\gamma_{i j_{p}}=\left(u_{i}-\alpha_{i}\right) \prod_{k=p+1}^{|J|}\left(1+u_{j_{k}}-\alpha_{j_{k}}\right) \text { for each } j_{p} \in J, p \neq|J| .
$$


Consequently,

$$
\sum_{k=j_{p}+1}^{i} \gamma_{i k}\left(u_{k}-\alpha_{k}\right)=\gamma_{i j_{p}}=\left(u_{i}-\alpha_{i}\right) \prod_{\substack{k=j_{p}+1: \\ \alpha_{k} \neq 0}}^{i-1}\left(1+u_{k}-\alpha_{k}\right) \text { for each } j_{p} \in J, p \neq|J|,
$$

with the first equation following from (6), and with the second equation following from (12) since $\alpha_{k}=0$ for all $k \in\left\{j_{p}+1, \cdots, i-1\right\}$ with $k \notin J$. Thus, for every $i \geq 2$, equation (10) holds for each $j>i$ with $\alpha_{j} \neq 0$, as every such $j$ has $j=j_{p}$ for some $p \in\{1, \cdots,|J|\}$. This completes the proof.

\section{Convex Hull Representation}

To establish that $\bar{F}=\operatorname{conv}(S)$, we begin with Lemma 4 below that identifies the inverse of a specially-structured $n \times n$ lower-triangular matrix. The form of this inverse will coincide with a subset of the coefficients $\gamma_{i j}$ found in the inequalities of (8) in such a manner that each row corresponds to a select inequality. As will be seen in the proof of upcoming Theorem 2, we need not consider the trivial case where $n=1$.

Lemma 4 Consider any $n \times n$ lower-triangular matrix $\boldsymbol{A}, n \geq 2$, having ones along the diagonal, and any $n-1$ scalars $r_{2}, \cdots, r_{n}$ such that the $(i, j)^{\text {th }}$ entry $a_{i j}$ has $a_{i j}=r_{i}$ for all $(i, j), j<i$. Then the inverse of $\boldsymbol{A}$, denoted by $\boldsymbol{A}^{-1}$, is that lower-triangular matrix whose $(i, j)^{\text {th }}$ entry $a_{i j}^{-1}$ is given by:

$$
a_{i j}^{-1}=\left\{\begin{array}{ll}
0 & \text { if } j>i \\
1 & \text { if } j=i \\
-r_{i} & \text { if } j=i-1 \\
-r_{i} \prod_{k=j+1}^{i-1}\left(1-r_{k}\right) & \text { if } j \leq i-2
\end{array} \quad \forall(i, j) .\right.
$$

Proof The matrix $\boldsymbol{A}^{-1}$ is trivially lower-triangular with ones along the diagonal. The proof is to show, for all $(i, j), j<i$, that the dot product of row $i$ of $\boldsymbol{A}$ with column $j$ of $\boldsymbol{A}^{-1}$ equals 0 ; that is, that:

$$
\sum_{k=j}^{i} a_{i k} a_{k j}^{-1}=0 \quad \forall(i, j), j<i
$$

From the definition of $\boldsymbol{A}$ and (14), we have that:

$$
\sum_{k=j}^{i} a_{i k} a_{k j}^{-1}=r_{i}(1)+(1)\left(-r_{i}\right)=0 \quad \forall(i, j), j=i-1,
$$

so that (15) holds true for all $(i, j), j=i-1$. To show that (15) holds true for all $(i, j), j \leq i-2$, and complete the proof, it is sufficient to show that:

$$
\sum_{k=j}^{p} a_{k j}^{-1}=\prod_{k=j+1}^{p}\left(1-r_{k}\right) \quad \forall(p, j), j \leq p-1 .
$$


Then we will have:

$$
\begin{aligned}
\sum_{k=j}^{i} a_{i k} a_{k j}^{-1} & =\sum_{k=j}^{i-1} a_{i k} a_{k j}^{-1}+a_{i i} a_{i j}^{-1}=r_{i} \sum_{k=j}^{i-1} a_{k j}^{-1}+a_{i j}^{-1} \\
& =r_{i} \prod_{k=j+1}^{i-1}\left(1-r_{k}\right)-r_{i} \prod_{k=j+1}^{i-1}\left(1-r_{k}\right)=0 \quad \forall(i, j), j \leq i-2,
\end{aligned}
$$

where the first and fourth equations are algebra, the second is by definition of $\boldsymbol{A}$, and the third is by (14) and by (16) with $p=i-1$. To establish (16), observe that these equations hold true for all $(p, j), j=p-1$, because $a_{(p-1)(p-1)}^{-1}+a_{p(p-1)}^{-1}=$ $1-r_{p}$ from (14). Using induction, assume that (16) holds true for all $(p, j), j \in$ $\{p-1, \cdots, p-t\}$, for some $t \in\{1, \cdots, p-2\}$, and consider (16) for $j=p-t-1$. We have:

$$
\sum_{k=j}^{p} a_{k j}^{-1}=\sum_{k=j}^{p-1} a_{k j}^{-1}+a_{p j}^{-1}=\prod_{k=j+1}^{p-1}\left(1-r_{k}\right)+\left[-r_{p} \prod_{k=j+1}^{p-1}\left(1-r_{k}\right)\right]=\prod_{k=j+1}^{p}\left(1-r_{k}\right),
$$

where the first and third equations are algebra and the second equation is due to the inductive hypothesis and the definition of $a_{p j}^{-1}$ in (14) with $j \leq p-2$. This completes the proof.

The example below illustrates this Lemma.

Example 2 Let $n=4$ and $\left(r_{2}, r_{3}, r_{4}\right)=(0,-4,-2)$. Then the lower-triangular matrices $\boldsymbol{A}$ and $\boldsymbol{A}^{-1}$ are given by: $\boldsymbol{A}=\left[\begin{array}{cccc}1 & 0 & 0 & 0 \\ 0 & 1 & 0 & 0 \\ -4 & -4 & 1 & 0 \\ -2 & -2 & -2 & 1\end{array}\right]$ and $\boldsymbol{A}^{-1}=\left[\begin{array}{cccc}1 & 0 & 0 & 0 \\ 0 & 1 & 0 & 0 \\ 4 & 4 & 1 & 0 \\ 10 & 10 & 2 & 1\end{array}\right]$.

Remark 2 below relates the matrix $\boldsymbol{A}^{-1}$ of Lemma 4 to the set $\bar{F}$ of (8).

Remark 2 Given any $k \in\{2, \cdots, p\}$, the inequalities:

$$
\begin{aligned}
& \sum_{\substack{j \leq i: \\
\alpha_{j}>0}} \gamma_{i j}\left(\alpha_{j}-x_{j}\right) \geq 0 \text { for all } i \in\{1, \cdots, k-1\} \text { such that } \alpha_{i}>0, \text { and } \\
& \sum_{\substack{j<k: \\
\alpha_{j}>0}} \gamma_{k j}\left(\alpha_{j}-x_{j}\right)+\left(\alpha_{k}-x_{k}\right) \geq 0
\end{aligned}
$$

found within $\bar{F}$ of (8), and expressible in matrix notation as:

$$
\Gamma\left(\boldsymbol{\alpha}^{\prime}-\boldsymbol{x}^{\prime}\right) \geq \mathbf{0}
$$

have, by (9) of Lemma 3, the matrix $\boldsymbol{\Gamma}$ of the form $\boldsymbol{A}^{-1}$ of Lemma 4 , where the inequality associated with each $i$ has $r_{i}=\left(\alpha_{i}-u_{i}\right)$. Here, the notation $\boldsymbol{\alpha}^{\prime}$ and $\boldsymbol{x}^{\prime}$ is used to denote those reduced versions of $\boldsymbol{\alpha}$ and $\boldsymbol{x}$, respectively, that include only the components $\alpha_{j}$ and $x_{j}$ for which $j<k$ and $\alpha_{j}>0$, or for which $j=k$. Such a representation is possible since, for each $i \leq k$, we have that $\gamma_{i j}=0$ for all $j<i$ with $\alpha_{j}=0$ by (9) (and equivalently by $(6)$ ). 
Example 3 Reconsider Example 1, and let $k=5$. Since $\alpha_{4}=0$, we have $\left(\boldsymbol{\alpha}^{\prime}\right)^{T}=$ $\left(\alpha_{1}, \alpha_{2}, \alpha_{3}, \alpha_{5}\right)=(3,4,1,2)$ and $\left(\boldsymbol{x}^{\prime}\right)^{T}=\left(x_{1}, x_{2}, x_{3}, x_{5}\right)$, so that (17) takes the form:

$$
\left[\begin{array}{cccc}
1 & 0 & 0 & 0 \\
0 & 1 & 0 & 0 \\
4 & 4 & 1 & 0 \\
10 & 10 & 2 & 1
\end{array}\right]\left[\begin{array}{l}
\left(3-x_{1}\right) \\
\left(4-x_{2}\right) \\
\left(1-x_{3}\right) \\
\left(2-x_{5}\right)
\end{array}\right] \geq\left[\begin{array}{l}
0 \\
0 \\
0 \\
0
\end{array}\right]
$$

with:

$$
\boldsymbol{\Gamma}^{-1}=\left[\begin{array}{cccc}
1 & 0 & 0 & 0 \\
\left(\alpha_{2}-u_{2}\right) & 1 & 0 & 0 \\
\left(\alpha_{3}-u_{3}\right) & \left(\alpha_{3}-u_{3}\right) & 1 & 0 \\
\left(\alpha_{5}-u_{5}\right) & \left(\alpha_{5}-u_{5}\right) & \left(\alpha_{5}-u_{5}\right) & 1
\end{array}\right]=\left[\begin{array}{cccc}
1 & 0 & 0 & 0 \\
0 & 1 & 0 & 0 \\
-4 & -4 & 1 & 0 \\
-2 & -2 & -2 & 1
\end{array}\right]
$$

as in Example 2.

We now present our main result.

Theorem 2 Given any $p \geq 1$, and any $\boldsymbol{\alpha} \in \mathbb{Z}_{\geq \mathbf{0}}^{p}$ and $\boldsymbol{u} \in \mathbb{Z}_{>\mathbf{0}}^{p}$ with $\boldsymbol{\alpha} \leq \boldsymbol{u}$ and $\alpha_{1}=u_{1}$, the sets $F$ and $\bar{F}$ defined in $(7)$ and $(8)$, respectively, have conv $(F)=\bar{F}$.

Proof The proof is to show that an arbitrarily-selected extreme point $\hat{\boldsymbol{x}}$ of the polytope $\bar{F}$ is integral. Let $K \subseteq\{1, \cdots, p\}$ denote the set of indices $k$ having $\alpha_{k}=u_{k}$ and $\sum_{j \leq k} \gamma_{k j}\left(\alpha_{j}-\hat{x}_{j}\right)=0$. Remark 1 gives us that $\hat{x}_{k}=u_{k}$ for all $k \in K$. Consequently, if $\sum_{j<k} \gamma_{k j}\left(\alpha_{j}-\hat{x}_{j}\right)>0$ for all $k \notin K$, so that none of the remaining $p-|K|$ structural inequalities in (8) are binding, we have that every entry of $\hat{\boldsymbol{x}}$ realizes a value at either its lower or upper bound, establishing the result. Otherwise, select that index $t \notin K$ having $\sum_{j \leq t} \gamma_{t j}\left(\alpha_{j}-\hat{x}_{j}\right)=0$ and $\sum_{j \leq k} \gamma_{k j}\left(\alpha_{j}-\hat{x}_{j}\right)>0$ for all $k \notin K, k>t$, so that:

$$
\alpha_{t}<u_{t} \text { and } \sum_{j \leq t} \gamma_{t j}\left(\alpha_{j}-\hat{x}_{j}\right)=0
$$

In other words, $t$ is the largest index $i$ not in the set $K$ such that the inequality $\sum_{j \leq i} \gamma_{i j}\left(\alpha_{j}-x_{j}\right) \geq 0$ of $(8)$ is satisfied with equality at $\hat{\boldsymbol{x}}$. Observe that $t \geq 2$ because $\alpha_{1}=u_{1}$ (which will prove consistent with $n \geq 2$ in Lemma 4). Form a reduced version of $\bar{F}$ by removing the last $(p-t)$ inequalities of (8), and by setting inequality $t$ to equality as in (18), to obtain:

$$
\left\{(\boldsymbol{x}, \boldsymbol{s}) \in \mathbb{R}_{\geq \mathbf{0}}^{p} \times \mathbb{R}_{\geq \mathbf{0}}^{t-1}: \boldsymbol{x} \leq \boldsymbol{u}, \sum_{j \leq i} \gamma_{i j}\left(\alpha_{j}-x_{j}\right)=s_{i}, i<t, \sum_{j \leq t} \gamma_{t j}\left(\alpha_{j}-x_{j}\right)=0\right\}
$$

where we have introduced a vector of slack variables $s \in \mathbb{R}_{>\mathbf{0}}^{t-1}$. Because the only possible constraints in the removed $(p-t)$ inequalities of $(8)$ that are binding at $\hat{\boldsymbol{x}}$ are also enforced by $\boldsymbol{x} \leq \boldsymbol{u}$ of (19), we have that $\hat{\boldsymbol{x}}$ is an extreme point of (19). Thus, the proof reduces to showing that the polytope (19) has all integral extreme points. Now, since no variable $x_{i}, i>t$, appears in any of the equality restrictions of (19), every extreme point to (19) must have every such $x_{i}$ at one of its bounds. 
Thus, we can eliminate all such $x_{i}$ from (19), so the proof is to show (with the truncated vector $\boldsymbol{x}$ ) that:

$$
\left\{(\boldsymbol{x}, \boldsymbol{s}) \in \mathbb{R}_{\geq \mathbf{0}}^{t} \times \mathbb{R}_{\geq \mathbf{0}}^{t-1}: \boldsymbol{x} \leq \boldsymbol{u}, \sum_{j \leq i} \gamma_{i j}\left(\alpha_{j}-x_{j}\right)=s_{i}, i<t, \sum_{j \leq t} \gamma_{t j}\left(\alpha_{j}-x_{j}\right)=0\right\}
$$

has all integral extreme points.

Permute the rows and columns of the equations of (20) so that the equations having $\alpha_{i}=0$ appear at the bottom, and so that the variables $x_{i}$ having $\alpha_{i}=0$ appear at the right. Upon so doing, the equations of (20) take the matrix form:

$$
\left[\begin{array}{c|c|c}
\boldsymbol{L} & \mathbf{0} & \mathbf{0} \\
\hline \boldsymbol{\gamma}_{t}^{\prime} & 1 & \mathbf{0} \\
\hline \boldsymbol{B} & \mathbf{0} & \boldsymbol{I}
\end{array}\right]\left[\begin{array}{c}
\left(\boldsymbol{\alpha}^{\prime}-\boldsymbol{x}^{\prime}\right) \\
\left(\alpha_{t}-x_{t}\right) \\
-\boldsymbol{x}^{\prime \prime}
\end{array}\right]=\left[\begin{array}{c}
\boldsymbol{s}^{\prime} \\
0 \\
\boldsymbol{s}^{\prime \prime}
\end{array}\right]
$$

using the following notation. Let $\tau \geq 1$ denote the number of indices $i$ having $\alpha_{i}>0, i<t$. The first column of the partitioned matrix of (21) corresponds to the $\tau$ variables $x_{i}$ having $\alpha_{i}>0, i<t$, so that $\boldsymbol{L}$ is the $\tau \times \tau$ lower-triangular matrix with ones along the diagonal associated with the equations having $\alpha_{i}>$ $0, i<t, \gamma_{t}^{\prime}$ is the $1 \times \tau$ row vector associated with equation $t$, and $\boldsymbol{B}$ is the $t-(\tau+1) \times \tau$ matrix associated with the equations having $\alpha_{i}=0, i<t$. In this manner, the vector $\left(\boldsymbol{\alpha}^{\prime}-\boldsymbol{x}^{\prime}\right)$ is associated with the first $\tau$ entries of $(\boldsymbol{\alpha}-\boldsymbol{x})$ having $\alpha_{i}>0, i<t$. The second and third columns of the partitioned matrix are associated with the expression $\left(\alpha_{t}-x_{t}\right)$ and the $t-(\tau+1)$ entries of $(\boldsymbol{\alpha}-\boldsymbol{x})$ having $\alpha_{i}=0, i<t$, respectively, so that the vector $-\boldsymbol{x}^{\prime \prime}$ represents these last $t-(\tau+1)$ expressions. Then the vectors $s^{\prime}$ and $s^{\prime \prime}$ represent the nonnegative slack variables on the first and last families of constraints, respectively. The matrix $\boldsymbol{I}$ and the matrices $\mathbf{0}$ denote the suitably-dimensioned identity matrix and matrices of all zeroes, respectively.

Remark 2 with $k=t$ gives us that the $(\tau+1) \times(\tau+1)$ submatrix $\left[\frac{\boldsymbol{L} \mid \mathbf{0}}{\boldsymbol{\gamma}_{t}^{\prime} \mid 1}\right]$ of $(21)$ takes the form $\boldsymbol{A}^{-1}$ of Lemma 4 , with $r_{i}=\alpha_{i}-u_{i}$ for all $i<t$ having $\alpha_{i}>0$, and with $r_{t}=\alpha_{t}-u_{t}$. Consequently, we can equivalently rewrite the first $\tau+1$ rows of (21) by left-multiplying the corresponding lower-triangular matrix $\boldsymbol{A}$ by these rows. Moreover, given any row $q$ of the matrix $\boldsymbol{B}$, say $\boldsymbol{b}_{q}$, corresponding to some $q<t$ with $\alpha_{q}=0$, we have by (6) that only the first $n_{q}$ entries of $\boldsymbol{b}_{q}$ have nonzero values, where $n_{q}$ is the number of indices $i$ having $\alpha_{i}>0$ and $i<q$. Construct the $\left(n_{q}+1\right) \times\left(n_{q}+1\right)$ submatrix of $(21)$ given by $\left[\begin{array}{l|l}\boldsymbol{L}_{q} & 0 \\ \hline \boldsymbol{b}_{q}^{\prime} & 1\end{array}\right]$, where $\boldsymbol{L}_{q}$ consists of the first $n_{q}$ rows and $n_{q}$ columns of $\boldsymbol{L}$, and where $\boldsymbol{b}_{q}^{\prime}$ consists of the first $n_{q}$ entries of $\boldsymbol{b}_{q}$. Then, for each such $\boldsymbol{b}_{q}$, Remark 2 with $k=n_{q}+1$ gives us that this matrix is invertible, with Lemma 4 establishing that the last row of the inverse matrix consists of $\left[-u_{q}, \cdots,-u_{q}, 1\right]$. Thus, for any such $\boldsymbol{b}_{q}$, we can add the scalar multiple $-u_{q}$ of the first $n_{q}$ rows of $\boldsymbol{L}$ to the associated row $\boldsymbol{b}_{q}$ within $\boldsymbol{B}$ of (21) to reduce $\boldsymbol{b}_{q}$ to a row of zeroes (as every entry in these $n_{q}$ rows of $\boldsymbol{L}$ that is not found within $\boldsymbol{L}_{q}$ must be 0 by (6)). Upon performing these two sets of operations, the below 
three families of equations result:

$$
\begin{aligned}
& x_{i}=\alpha_{i}+\left(u_{i}-\alpha_{i}\right)\left(\sum_{\substack{j<i: \\
\alpha_{j}>0}} s_{j}\right)-s_{i} \text { for all } i=1, \cdots, t-1 \text { such that } \alpha_{i}>0, \\
& x_{t}=\alpha_{t}+\left(u_{t}-\alpha_{t}\right)\left(\sum_{\substack{j<t: \\
\alpha_{j}>0}} s_{j}\right), \text { and } \\
& x_{i}=u_{i}\left(\sum_{\substack{j<i: \\
\alpha_{j}>0}} s_{j}\right)-s_{i} \text { for all } i=2, \cdots, t-1 \text { such that } \alpha_{i}=0 .
\end{aligned}
$$

Then (20) becomes:

$$
\left\{(\boldsymbol{x}, \boldsymbol{s}) \in \mathbb{R}_{\geq \mathbf{0}}^{t} \times \mathbb{R}_{\geq \mathbf{0}}^{t-1}: \boldsymbol{x} \leq \boldsymbol{u}, \quad(22),(23),(24)\right\}
$$

Since $\alpha_{t}<u_{t}$ by (18), equation (23) in (25) is equivalent to:

$$
\sum_{\substack{j<t: \\ \alpha_{j}>0}} s_{j}+\frac{u_{t}-x_{t}}{u_{t}-\alpha_{t}}=1
$$

To show that (25) has all integral extreme points and complete the proof, we first show that the set (27) below obtained from (25) by removing (22) and all associated variables $\boldsymbol{x}^{\prime}$, and by replacing (23) by (26), has all integral extreme points.

$$
\left\{\left(x_{t}, \boldsymbol{x}^{\prime \prime}, \boldsymbol{s}\right) \in \mathbb{R}^{1} \times \mathbb{R}_{\geq \mathbf{0}}^{t-(\tau+1)} \times \mathbb{R}_{\geq \mathbf{0}}^{t-1}: \alpha_{t} \leq x_{t} \leq u_{t}, \boldsymbol{x}^{\prime \prime} \leq \boldsymbol{u}^{\prime \prime},(24),(26)\right\}
$$

Here, we have used in (27) that $\alpha_{t} \leq x_{t}$ for $\left(x_{t}, \boldsymbol{s}^{\prime}\right)$ satisfying $\boldsymbol{s}^{\prime} \geq \mathbf{0}$ and (26). Toward this end, let:

$$
y_{i}=\left\{\begin{array}{ll}
\frac{x_{i}}{u_{i}} & \text { if } \alpha_{i}=0 \\
\frac{x_{t}-\alpha_{t}}{u_{t}-\alpha_{t}} & \text { if } i=t
\end{array} \quad \text { for all } i \text { such that } \alpha_{i}=0 \text { or } i=t,\right.
$$

so that the inverse transformation is given by:

$$
x_{i}=\left\{\begin{array}{ll}
u_{i} y_{i} & \text { if } \alpha_{i}=0 \\
y_{t}\left(u_{t}-\alpha_{t}\right)+\alpha_{t} & \text { if } i=t
\end{array} \quad \text { for all } i \text { such that } \alpha_{i}=0 \text { or } i=t .\right.
$$

Then the substitution (28) transforms (27) to:

$$
\left\{\begin{array}{l}
\left(\boldsymbol{y}, \boldsymbol{s}^{\prime}\right) \in \mathbb{R}_{\geq \mathbf{0}}^{t-\tau} \times \mathbb{R}_{\geq \mathbf{0}}^{\tau}: \boldsymbol{y} \leq \mathbf{1}, \\
y_{i} \leq \sum_{\substack{j<i: \\
\alpha_{j}>0}} s_{j} \text { for all } i=2, \cdots, t-1 \text { such that } \alpha_{i}=0, \\
y_{t}=\sum_{\substack{j<t: \\
\alpha_{j}>0}} s_{j}
\end{array}\right\},
$$


where we have removed the nonnegative slack variables $\boldsymbol{s}^{\prime \prime}$ by writing (24) as inequality restrictions. As each variable within $\boldsymbol{s}^{\prime}$ of (30) satisfies the "consecutive ones" property ([7, page 544, Definition 2.2] or [10]) mentioned in Section 1, and as each variable within $\boldsymbol{y}$ appears exactly once, exclusive of the bounds $\mathbf{0} \leq \boldsymbol{y} \leq \mathbf{1}$, the polytope (30) has all binary extreme points. The inverse transformation (29) then gives us that (27) has all integral extreme points. Finally, consider (25) and observe that equations (22) of (25) uniquely define each variable $x_{i}$ of $\boldsymbol{x}^{\prime}$ in terms of $\boldsymbol{s}^{\prime}$, and these $x_{i}$ values satisfy $\mathbf{0} \leq \boldsymbol{x}^{\prime} \leq \boldsymbol{u}^{\prime}$ for every $\boldsymbol{s}$ such that $\left(x_{t}, \boldsymbol{x}^{\prime \prime}, \boldsymbol{s}\right)$ is feasible to (27), as every such $x_{i}$ realizes one of the three values $\left(\alpha_{i}-1\right), \alpha_{i}$, or $u_{i}$ at each extreme point. This conclusion follows because the $y_{t}=\sum_{\substack{j<t: \\ \alpha_{j}>0}} s_{j}, \boldsymbol{y}$ binary, $\boldsymbol{s}^{\prime} \geq \mathbf{0}$, extreme point characterization of (27) permits at most one entry of $\boldsymbol{s}^{\prime}$ to equal to 1 , and forces the remaining entries to equal to 0 . The proof is complete.

In addition to characterizing the set $\bar{F}$ of (8) as having all integral extreme points, the above proof provides insights into the polyhedral structure of (20) and (25), as well as $\bar{F}$ itself. First, the bounds $\mathbf{0} \leq \boldsymbol{x}^{\prime} \leq \boldsymbol{u}^{\prime}$ can be deleted in both (20) and (25), as demonstrated at the conclusion of the proof. Second, when $\boldsymbol{\alpha}>\mathbf{0}$, there are no equations present within (24), so that $t=\tau+1$ and (30) reduces to:

$$
\left\{\left(y_{t}, \boldsymbol{s}^{\prime}\right) \in \mathbb{R}_{\geq \mathbf{0}}^{1} \times \mathbb{R}_{\geq \mathbf{0}}^{t-1}: y_{t} \leq 1, y_{t}=\sum_{\substack{j<t: \\ \alpha_{j}>0}} s_{j}\right\},
$$

which has the $t$ extreme points:

$$
\left[\begin{array}{c}
s_{1}^{\prime} \\
s_{2}^{\prime} \\
\vdots \\
s_{\tau}^{\prime} \\
y_{t}
\end{array}\right]=\left\{\left[\begin{array}{c}
1 \\
0 \\
\vdots \\
0 \\
1
\end{array}\right],\left[\begin{array}{c}
0 \\
1 \\
\vdots \\
0 \\
1
\end{array}\right], \cdots,\left[\begin{array}{c}
0 \\
0 \\
\vdots \\
1 \\
1
\end{array}\right],\left[\begin{array}{c}
0 \\
0 \\
\vdots \\
0 \\
0
\end{array}\right]\right\}
$$

Then the $y_{t}=\frac{x_{t}-\alpha_{t}}{u_{t}-\alpha_{t}}$ transformation of (28), together with equations (22) of (25), define $(20)$ and $(25)$ as the $(t-1)$-dimensional simplex in $\mathbb{R}^{t}$ whose $t$ extreme points are given by:

$$
\left[\begin{array}{c}
x_{1} \\
x_{2} \\
x_{3} \\
\vdots \\
x_{t}
\end{array}\right]=\left\{\left[\begin{array}{c}
\alpha_{1}-1 \\
u_{2} \\
u_{3} \\
\vdots \\
u_{t}
\end{array}\right],\left[\begin{array}{c}
\alpha_{1} \\
\alpha_{2}-1 \\
u_{3} \\
\vdots \\
u_{t}
\end{array}\right], \cdots,\left[\begin{array}{c}
\alpha_{1} \\
\alpha_{2} \\
\vdots \\
\alpha_{t-1}-1 \\
u_{t}
\end{array}\right],\left[\begin{array}{c}
\alpha_{1} \\
\alpha_{2} \\
\vdots \\
\alpha_{t-1} \\
\alpha_{t}
\end{array}\right]\right\} .
$$

These extreme points are consistent with the result in the proof that, at every extreme point to (25), each variable $x_{i}$ of $\boldsymbol{x}^{\prime}$ must realize one of the three values $\left(\alpha_{i}-1\right), \alpha_{i}$, or $u_{i}$. Third, when $\alpha_{i}=0$ for some $i$, the associated $x_{i}$ must have $x_{i}=0$ or $x_{i}=u_{i}$ at every extreme point to $\bar{F}$. For the case in which $i>t$, the initial part of the proof establishes this result via (19), while for the case in which $i<t$, the transformation (29) gives this same result due to the binary nature of the extreme points to (30).

Theorem 2 and its proof are demonstrated in Example 4 below. 
Example 4 As in Example 1, let $p=5, \boldsymbol{\alpha}^{T}=(3,4,1,0,2)$, and $\boldsymbol{u}^{T}=(3,4,5,3,4)$. The corresponding set $\bar{F}$ of (8) is given by:

$$
\bar{F}=\left\{\boldsymbol{x} \in \mathbb{R}_{\geq \mathbf{0}}^{5}: \boldsymbol{x} \leq \boldsymbol{u},\left[\begin{array}{lllll}
1 & 0 & 0 & 0 & 0 \\
0 & 1 & 0 & 0 & 0 \\
4 & 4 & 1 & 0 & 0 \\
15 & 15 & 3 & 1 & 0 \\
10 & 10 & 2 & 0 & 1
\end{array}\right]\left[\begin{array}{c}
\left(3-x_{1}\right) \\
\left(4-x_{2}\right) \\
\left(1-x_{3}\right) \\
\left(0-x_{4}\right) \\
\left(2-x_{5}\right)
\end{array}\right] \geq\left[\begin{array}{l}
0 \\
0 \\
0 \\
0 \\
0
\end{array}\right]\right\} .
$$

Suppose that the fifth inequality is satisfied with equality at some extreme point $\hat{\boldsymbol{x}}$ so that $t=5$ as found in (18), with $\tau=3$, consistent with the proof of Theorem 2 . The resulting system (20), upon including the slack variables $\boldsymbol{s} \in \mathbb{R}_{\geq \mathbf{0}}^{4}$ as in (20), can be expressed in matrix notation, as in (21), by:

$$
\left\{(\boldsymbol{x}, \boldsymbol{s}) \in \mathbb{R}_{\geq \mathbf{0}}^{5} \times \mathbb{R}_{\geq \mathbf{0}}^{4}: \boldsymbol{x} \leq \boldsymbol{u},\left[\begin{array}{ccc|c|c}
1 & 0 & 0 & 0 & 0 \\
0 & 1 & 0 & 0 & 0 \\
4 & 4 & 1 & 0 & 0 \\
\hline 10 & 10 & 2 & 1 & 0 \\
\hline 15 & 15 & 3 & 0 & 1
\end{array}\right]\left[\begin{array}{c}
\left(3-x_{1}\right) \\
\left(4-x_{2}\right) \\
\left(1-x_{3}\right) \\
\left(2-x_{5}\right) \\
-x_{4}
\end{array}\right]=\left[\begin{array}{c}
s_{1} \\
s_{2} \\
s_{3} \\
0 \\
s_{4}
\end{array}\right]\right\} .
$$

Adding $-u_{4}=-3$ times the sum of the first three equations to the fifth, and then multiplying the first four equations by the inverse matrix $\boldsymbol{\Gamma}^{-1}$ of Example 3, transforms (32) to the form of (25) given below:

$$
\left\{(\boldsymbol{x}, \boldsymbol{s}) \in \mathbb{R}_{\geq \mathbf{0}}^{5} \times \mathbb{R}_{\geq \mathbf{0}}^{4}: \boldsymbol{x} \leq \boldsymbol{u},\left[\begin{array}{l}
x_{1} \\
x_{2} \\
x_{3} \\
x_{5} \\
x_{4}
\end{array}\right]=\left[\begin{array}{l}
3 \\
4 \\
1 \\
2 \\
0
\end{array}\right]+\left[\begin{array}{ccccc}
-1 & 0 & 0 & 0 & 0 \\
0 & -1 & 0 & 0 & 0 \\
4 & 4 & -1 & 0 & 0 \\
2 & 2 & 2 & -1 & 0 \\
3 & 3 & 3 & 0 & -1
\end{array}\right]\left[\begin{array}{c}
s_{1} \\
s_{2} \\
s_{3} \\
0 \\
s_{4}
\end{array}\right]\right\}
$$

The system (27) with $\boldsymbol{x}^{\prime \prime}=x_{4}$ takes the form:

$$
\begin{aligned}
\left\{\left(x_{5}, x_{4}, s\right) \in \mathbb{R}^{1} \times \mathbb{R}_{\geq \mathbf{0}}^{1} \times \mathbb{R}_{\geq \mathbf{0}}^{4}:\right. & 2 \leq x_{5} \leq 4, x_{4} \leq 3, \\
& x_{4}=3\left(s_{1}+s_{2}+s_{3}\right)-s_{4}, \\
& \left.s_{1}+s_{2}+s_{3}+\frac{4-x_{5}}{2}=1\right\} .
\end{aligned}
$$

The transformation $y_{4}=\frac{x_{4}}{3}$ and $y_{5}=\frac{x_{5}-2}{2}$ of (28) changes (34), upon removing the nonnegative slack variable $s_{4}$, to the form:

$$
\left\{\left(\boldsymbol{y}, \boldsymbol{s}^{\prime}\right) \in \mathbb{R}_{\geq \mathbf{0}}^{2} \times \mathbb{R}_{\geq \mathbf{0}}^{3}: y_{4} \leq 1, y_{5} \leq 1, y_{4} \leq s_{1}+s_{2}+s_{3}, y_{5}=s_{1}+s_{2}+s_{3}\right\},
$$

as found in (30). This last set has the "consecutive ones" property, so that it has all binary extreme points. The extreme points are readily verified to be:

$$
\left[\begin{array}{l}
y_{4} \\
y_{5} \\
s_{1} \\
s_{2} \\
s_{3}
\end{array}\right]=\left\{\left[\begin{array}{l}
0 \\
0 \\
0 \\
0 \\
0
\end{array}\right],\left[\begin{array}{l}
0 \\
1 \\
1 \\
0 \\
0
\end{array}\right],\left[\begin{array}{l}
1 \\
1 \\
1 \\
0 \\
0
\end{array}\right],\left[\begin{array}{l}
0 \\
1 \\
0 \\
1 \\
0
\end{array}\right],\left[\begin{array}{l}
1 \\
1 \\
0 \\
1 \\
0
\end{array}\right],\left[\begin{array}{l}
0 \\
1 \\
0 \\
0 \\
1
\end{array}\right],\left[\begin{array}{l}
1 \\
1 \\
0 \\
0 \\
1
\end{array}\right]\right\},
$$

which, via the inverse transformation $x_{4}=3 y_{4}$ and $x_{5}=2 y_{5}+2$ of (29), gives the extreme points of (34) as:

$$
\left[\begin{array}{l}
x_{4} \\
x_{5} \\
s_{1} \\
s_{2} \\
s_{3}
\end{array}\right]=\left\{\left[\begin{array}{l}
0 \\
2 \\
0 \\
0 \\
0
\end{array}\right],\left[\begin{array}{l}
0 \\
4 \\
1 \\
0 \\
0
\end{array}\right],\left[\begin{array}{l}
3 \\
4 \\
1 \\
0 \\
0
\end{array}\right],\left[\begin{array}{l}
0 \\
4 \\
0 \\
1 \\
0
\end{array}\right],\left[\begin{array}{l}
3 \\
4 \\
0 \\
1 \\
0
\end{array}\right],\left[\begin{array}{l}
0 \\
4 \\
0 \\
0 \\
1
\end{array}\right],\left[\begin{array}{l}
3 \\
4 \\
0 \\
0 \\
1
\end{array}\right]\right\}
$$


Then, by (33), the extreme points to (32) in terms of $\left(x_{1}, x_{2}, x_{3}, x_{4}, x_{5}\right)$ are:

$$
\left[\begin{array}{l}
x_{1} \\
x_{2} \\
x_{3} \\
x_{4} \\
x_{5}
\end{array}\right]=\left\{\left[\begin{array}{l}
3 \\
4 \\
1 \\
0 \\
2
\end{array}\right],\left[\begin{array}{l}
2 \\
4 \\
5 \\
0 \\
4
\end{array}\right],\left[\begin{array}{l}
2 \\
4 \\
5 \\
3 \\
4
\end{array}\right],\left[\begin{array}{l}
3 \\
3 \\
5 \\
0 \\
4
\end{array}\right],\left[\begin{array}{l}
3 \\
3 \\
5 \\
3 \\
4
\end{array}\right],\left[\begin{array}{l}
3 \\
4 \\
0 \\
0 \\
4
\end{array}\right],\left[\begin{array}{l}
3 \\
4 \\
0 \\
3 \\
4
\end{array}\right]\right\}
$$

We conclude this section with a final example that considers the set $\bar{F}$ of (8) when $\boldsymbol{u}$ is the $p$-dimensional vector of ones and $\boldsymbol{\alpha} \in \mathbb{Z}^{p}$ is a binary vector. Thus, this example constructs the convex hull of a special instance of $S$ in (1) relative to the set of binary vectors that is lexicographically less than or equal to $\boldsymbol{\alpha}$. This special case having $\boldsymbol{u}=\mathbf{1}$ was studied in $[3,5,6]$. Notable simplifications to Theorem 2 for this case are: all coefficients $\gamma_{i j}$ are 0 or 1 by (6) and (9), $\boldsymbol{L}$ in (21) is an identity matrix due to the conditions of Remark 1 being satisfied for all $i$ having $\alpha_{i}=1, \gamma_{t}^{\prime}$ in (21) consists of all ones by (6) and (9) since the constraint index $t$ of the proof must have $\alpha_{t}=0$ by (18) and since the first $\tau$ rearranged variables have $\alpha_{i}=1$, and the transformations of (28) and (29) set $y_{i}=x_{i}$ for all $i$ such that $\alpha_{i}=0$ or $i=t$.

Example 5 Let $p=5, \boldsymbol{\alpha}^{T}=(1,0,1,1,0)$, and $\boldsymbol{u}^{T}=(1,1,1,1,1)$. The set $\bar{F}$ of $(8)$ is given by:

$$
\bar{F}=\left\{\boldsymbol{x} \in \mathbb{R}_{\geq \mathbf{0}}^{5}: \boldsymbol{x} \leq \mathbf{1},\left[\begin{array}{lllll}
1 & 0 & 0 & 0 & 0 \\
1 & 1 & 0 & 0 & 0 \\
0 & 0 & 1 & 0 & 0 \\
0 & 0 & 0 & 1 & 0 \\
1 & 0 & 1 & 1 & 1
\end{array}\right]\left[\begin{array}{l}
\left(1-x_{1}\right) \\
\left(0-x_{2}\right) \\
\left(1-x_{3}\right) \\
\left(1-x_{4}\right) \\
\left(0-x_{5}\right)
\end{array}\right] \geq\left[\begin{array}{l}
0 \\
0 \\
0 \\
0 \\
0
\end{array}\right]\right\},
$$

where $\mathbf{1} \in \mathbb{R}^{5}$ denotes the vector of ones. Suppose that the fifth inequality is satisfied with equality at some extreme point $\hat{\boldsymbol{x}}$ so that $t=5$ as found in (18), with $\tau=3$. System (20), expressed using (21), becomes:

$$
\left\{(\boldsymbol{x}, \boldsymbol{s}) \in \mathbb{R}_{\geq \mathbf{0}}^{5} \times \mathbb{R}_{\geq \mathbf{0}}^{4}: \boldsymbol{x} \leq \mathbf{1},\left[\begin{array}{lll|l|l}
1 & 0 & 0 & 0 & 0 \\
0 & 1 & 0 & 0 & 0 \\
0 & 0 & 1 & 0 & 0 \\
\hline 1 & 1 & 1 & 1 & 0 \\
\hline 1 & 0 & 0 & 0 & 1
\end{array}\right]\left[\begin{array}{c}
\left(1-x_{1}\right) \\
\left(1-x_{3}\right) \\
\left(1-x_{4}\right) \\
-x_{5} \\
-x_{2}
\end{array}\right]=\left[\begin{array}{c}
s_{1} \\
s_{3} \\
s_{4} \\
0 \\
s_{2}
\end{array}\right]\right\}
$$

Adding $-u_{2}=-1$ times the first equation to the fifth, and then multiplying (as per Lemma 4 and Remark 2 with $r_{2}=r_{3}=0$ and $r_{4}=-1$ ) the first four equations by $\left[\begin{array}{llll}1 & 0 & 0 & 0 \\ 0 & 1 & 0 & 0 \\ 0 & 0 & 1 & 0 \\ 1 & 1 & 1 & 1\end{array}\right]^{-1}=\left[\begin{array}{cccc}1 & 0 & 0 & 0 \\ 0 & 1 & 0 & 0 \\ 0 & 0 & 1 & 0 \\ -1 & -1 & -1 & 1\end{array}\right]$, transforms (36) to the form of $(25)$ given by:

$$
\left\{(\boldsymbol{x}, \boldsymbol{s}) \in \mathbb{R}_{\geq \mathbf{0}}^{5} \times \mathbb{R}_{\geq \mathbf{0}}^{4}: \boldsymbol{x} \leq \mathbf{1},\left[\begin{array}{l}
x_{1} \\
x_{3} \\
x_{4} \\
x_{5} \\
x_{2}
\end{array}\right]=\left[\begin{array}{l}
1 \\
1 \\
1 \\
0 \\
0
\end{array}\right]+\left[\begin{array}{ccccc}
-1 & 0 & 0 & 0 & 0 \\
0 & -1 & 0 & 0 & 0 \\
0 & 0 & -1 & 0 & 0 \\
1 & 1 & 1 & -1 & 0 \\
1 & 0 & 0 & 0 & -1
\end{array}\right]\left[\begin{array}{l}
s_{1} \\
s_{3} \\
s_{4} \\
0 \\
s_{2}
\end{array}\right]\right\}
$$

Then (27) with $\boldsymbol{x}^{\prime \prime}=x_{2}$ takes the form:

$$
\begin{aligned}
\left\{\left(x_{5}, x_{2}, \boldsymbol{s}\right) \in \mathbb{R}^{1} \times \mathbb{R}_{\geq \mathbf{0}}^{1} \times \mathbb{R}_{\geq \mathbf{0}}^{4}:\right. & 0 \leq x_{5} \leq 1, x_{2} \leq 1, \\
& x_{2}=s_{1}-s_{2}, \\
& \left.s_{1}+s_{3}+s_{4}+\left(1-x_{5}\right)=1\right\} .
\end{aligned}
$$


The transformation $y_{2}=x_{2}$ and $y_{5}=x_{5}$ of (28) changes (38), upon removing the nonnegative slack variable $s_{2}$, to the form:

$$
\left\{\left(\boldsymbol{y}, \boldsymbol{s}^{\prime}\right) \in \mathbb{R}_{\geq \mathbf{0}}^{2} \times \mathbb{R}_{\geq \mathbf{0}}^{3}: y_{2} \leq 1, y_{5} \leq 1, y_{2} \leq s_{1}, y_{5}=s_{1}+s_{3}+s_{4}\right\},
$$

as found in (30). This last set has the binary extreme points given by:

$$
\left[\begin{array}{l}
y_{2} \\
y_{5} \\
s_{1} \\
s_{3} \\
s_{4}
\end{array}\right]=\left\{\left[\begin{array}{l}
0 \\
0 \\
0 \\
0 \\
0
\end{array}\right],\left[\begin{array}{l}
0 \\
1 \\
1 \\
0 \\
0
\end{array}\right],\left[\begin{array}{l}
1 \\
1 \\
1 \\
0 \\
0
\end{array}\right],\left[\begin{array}{l}
0 \\
1 \\
0 \\
1 \\
0
\end{array}\right],\left[\begin{array}{l}
0 \\
1 \\
0 \\
0 \\
1
\end{array}\right]\right\} .
$$

The inverse transformation $x_{2}=y_{2}$ and $x_{5}=y_{5}$ of (29) gives the extreme points of (38) to be:

$$
\left[\begin{array}{l}
x_{2} \\
x_{5} \\
s_{1} \\
s_{3} \\
s_{4}
\end{array}\right]=\left\{\left[\begin{array}{l}
0 \\
0 \\
0 \\
0 \\
0
\end{array}\right],\left[\begin{array}{l}
0 \\
1 \\
1 \\
0 \\
0
\end{array}\right],\left[\begin{array}{l}
1 \\
1 \\
1 \\
0 \\
0
\end{array}\right],\left[\begin{array}{l}
0 \\
1 \\
0 \\
1 \\
0
\end{array}\right],\left[\begin{array}{l}
0 \\
1 \\
0 \\
0 \\
1
\end{array}\right]\right\} .
$$

Then (37) gives the extreme points to (36) in terms of $\left(x_{1}, x_{2}, x_{3}, x_{4}, x_{5}\right)$ as:

$$
\left[\begin{array}{l}
x_{1} \\
x_{2} \\
x_{3} \\
x_{4} \\
x_{5}
\end{array}\right]=\left\{\left[\begin{array}{l}
1 \\
0 \\
1 \\
1 \\
0
\end{array}\right],\left[\begin{array}{l}
0 \\
0 \\
1 \\
1 \\
1
\end{array}\right],\left[\begin{array}{l}
0 \\
1 \\
1 \\
1 \\
1
\end{array}\right],\left[\begin{array}{l}
1 \\
0 \\
0 \\
1 \\
1
\end{array}\right],\left[\begin{array}{l}
1 \\
0 \\
1 \\
0 \\
1
\end{array}\right]\right\} .
$$

\section{Conclusions and Extensions to Integral Knapsack Polytopes}

This paper provides an explicit algebraic description of the convex hull of bounded, nonnegative integer vectors that are lexicographically less-than-or-equal-to a given vector. The number of required inequalities is linear in the number of vector entries. This work generalizes related results of $[2,3,5,8]$ that consider, in different contexts, similar characterizations for binary vectors.

Building upon an observation of [6] that relates lexicographic orderings of binary variables with $0-1$ knapsack polytopes having "weakly super-decreasing" coefficients, we conclude this paper by identifying the convex hull of certain speciallystructured integral knapsack polytopes. A set of scalars $a_{1}, a_{2}, \cdots, a_{p}$ is said to be weakly super-decreasing if:

$$
a_{j} \geq \sum_{i=j+1}^{p} a_{i} \text { for each } j=1, \cdots, p-1 .
$$

Consider a binary knapsack polytope:

$$
B K P(\boldsymbol{x}) \equiv\left\{\boldsymbol{x} \in \mathbb{Z}_{\geq \mathbf{0}}^{p}: \boldsymbol{x} \leq \mathbf{1}, \sum_{j=1}^{p} a_{j} x_{j} \leq b\right\},
$$

where the coefficients $a_{j}$ are weakly super-decreasing. Select the largest scalar $b^{\prime} \leq b$ such that there exists an $\hat{\boldsymbol{x}} \in \mathbb{Z}_{\geq \mathbf{0}}^{p}, \hat{\boldsymbol{x}} \leq \mathbf{1}$, with $b^{\prime}=\sum_{j=1}^{p} a_{j} \hat{x}_{j}$, and let $\boldsymbol{\alpha}=\hat{\boldsymbol{x}}$. 
(Two possible such vectors $\hat{\boldsymbol{x}}$ can exist and, if so, choose the lexicographically larger.) Then a special (one-sided) case of [6] rewrites the polytope (41) as:

$$
B K P(\boldsymbol{x})=\left\{\boldsymbol{x} \in \mathbb{Z}_{\geq \mathbf{0}}^{p}: \boldsymbol{x} \leq \mathbf{1}, \boldsymbol{x} \preceq \boldsymbol{\alpha}\right\},
$$

so that (42) with $\boldsymbol{x} \in \mathbb{Z}_{\geq \mathbf{0}}^{p}$ and $\boldsymbol{x} \preceq \boldsymbol{\alpha}$ replaced by $\boldsymbol{x} \in \mathbb{R}_{\geq \mathbf{0}}^{p}$ and minimal cover inequalities of the form (3), respectively, gives the convex hull of (41) and (42).

Section 3 allows us to generalize this result to knapsack polytopes having bounded integer variables. As in (1), let $\boldsymbol{u} \in \mathbb{Z}_{\geq \mathbf{0}}^{p}$ denote a vector of upper bounds on $\boldsymbol{x} \in \mathbb{Z}_{\geq \mathbf{0}}^{p}$. Define scalars $a_{1}, a_{2}, \cdots, a_{p}$ to be "weakly super-decreasing relative to $\boldsymbol{u}^{\prime}$ if:

$$
a_{j} \geq \sum_{i=j+1}^{p} a_{i} u_{i} \text { for each } j=1, \cdots, p-1,
$$

so that (40) is that special case of (43) obtained by fixing $\boldsymbol{u}=\mathbf{1}$. Now, consider an integer knapsack polytope:

$$
I K P(\boldsymbol{x}) \equiv\left\{\boldsymbol{x} \in \mathbb{Z}_{\geq \mathbf{0}}^{p}: \boldsymbol{x} \leq \boldsymbol{u}, \sum_{j=1}^{p} a_{j} x_{j} \leq b\right\},
$$

where the coefficients $a_{j}$ satisfy (43). Again select the largest scalar $b^{\prime} \leq b$ such that there exists a $\hat{\boldsymbol{x}} \in \mathbb{Z}_{\geq \mathbf{0}}^{p}$ with $b^{\prime}=\sum_{j=1}^{p} a_{j} \hat{x}_{j}$, but this time allow $\hat{\boldsymbol{x}} \leq \boldsymbol{u}$. (As before, if two such vectors $\hat{\boldsymbol{x}}$ exist, choose the lexicographically larger.) Then, by setting $\boldsymbol{\alpha}=\hat{\boldsymbol{x}}$, we can rewrite (44) as:

$$
I K P(\boldsymbol{x})=\left\{\boldsymbol{x} \in \mathbb{Z}_{\geq \mathbf{0}}^{p}: \boldsymbol{x} \leq \boldsymbol{u}, \boldsymbol{x} \preceq \boldsymbol{\alpha}\right\},
$$

so that the set $\bar{F}$ of (8) gives, via Theorem 2 , the convex hull of (44) and (45).

Example 6 Consider the integer knapsack polytope in $p=5$ variables having $\boldsymbol{u}^{T}=$ $(3,4,5,3,4)$ given by:

$$
\left\{\boldsymbol{x} \in \mathbb{Z}_{\geq \mathbf{0}}^{5}: \boldsymbol{x} \leq \boldsymbol{u}, 480 x_{1}+96 x_{2}+16 x_{3}+4 x_{4}+x_{5} \leq 1842\right\},
$$

so that $b^{\prime}=b=1842$ and $\boldsymbol{\alpha}^{T}=(3,4,1,0,2)$, with the coefficients $\left(a_{1}, a_{2}, a_{3}, a_{4}, a_{5}\right)=$ $(480,96,16,4,1)$ weakly super-decreasing relative to $\boldsymbol{u}$. Thus the vectors $\boldsymbol{\alpha}$ and $\boldsymbol{u}$ are as given in Example 1 . Here, the $a_{j}$ were chosen to have $a_{5}=1$ and the four inequalities of (40) satisfied with equality. The inequalities below, taken from Example 1, define the convex hull of this polytope:

$$
\left\{\boldsymbol{x} \in \mathbb{R}_{\geq \mathbf{0}}^{5}: \boldsymbol{x} \leq \boldsymbol{u},\left[\begin{array}{ccccc}
1 & 0 & 0 & 0 & 0 \\
0 & 1 & 0 & 0 & 0 \\
4 & 4 & 1 & 0 & 0 \\
15 & 15 & 3 & 1 & 0 \\
10 & 10 & 2 & 0 & 1
\end{array}\right]\left[\begin{array}{l}
\left(3-x_{1}\right) \\
\left(4-x_{2}\right) \\
\left(1-x_{3}\right) \\
\left(0-x_{4}\right) \\
\left(2-x_{5}\right)
\end{array}\right] \geq\left[\begin{array}{l}
0 \\
0 \\
0 \\
0 \\
0
\end{array}\right]\right\}
$$

\section{Acknowledgements}

The investigator Warren Adams is grateful to the National Science Foundation for partially supporting this research under grant number CMMI-0968909. 


\section{References}

1. Angulo, G., Ahmed, S., and Dey, S.S., "Forbidding Extreme Points from the 0-1 Hypercube," Optimization Online, May 10, 2012. URL: http://www.optimizationonline.org/DB_HTML/2012/05/3463.html.

2. D'Andreagiovanni, F., "Pure 0-1 Programming Approaches to Wireless Network Design," Ph.D. Dissertation, University of Rome, 2010.

3. Gillmann, R. and Kaibel, V., "Revlex-initial 0/1-polytopes," Journal of Combinatorial Theory Series A, Vol. 113, No. 5, pp 799-821, 2006.

4. Gupte, A., "Convex Hulls of Superincreasing Knapsacks and Lexicographic Orderings", Optimization Online, December 4, 2012 (updated March 31, 2015). URL: http://www.optimization-online.org/DB_HTML/2012/12/3701.html

5. Laurent, M. and Sassano, A., "A Characterization of Knapsacks with the Max-Flow-MinCut Property," Operations Research Letters, Vol. 11, No. 2, pp 105-110, 1992.

6. Muldoon, F.M., Adams, W.P., and Sherali, H.D., "Ideal Representations of Lexicographic Orderings and Base-2 Expansions of Integer Variables," Operations Research Letters, Vol. 41, Issue 1, pp 32-39, 2013.

7. Nemhauser, G.L. and Wolsey, L.A., Integer and Combinatorial Optimization, John Wiley \& Sons, New York, NY, 1999.

8. Seymour, P.D., "The Matroids with the Max-Flow-Min-Cut Property," Journal of Combinatorial Theory Series B, Vol. 23, pp 189-222, 1977.

9. Shen, R., "Convex Hull Characterization of Special Polytopes in n-ary Variables," M.S. Thesis, Department of Mathematical Sciences, Clemson University, Clemson, SC, 2012.

10. Veinnott, Jr., A.F. and Wagner, H.M., "Optimal Capacity Scheduling - I," Operations Research, Vol. 10, No. 4, pp 518-532, 1962. 